\title{
Late-onset Tay-Sachs disease: Phenotypic characterization and genotypic correlations in 21 affected patients
}

Orit Neudorfer ${ }^{1}$, Gregory M. Pastores ${ }^{1,2}$, Bai J. Zeng ${ }^{1}$, John Gianutsos ${ }^{3}$, Charles M. Zarofft, and Edwin H. Kolodny ${ }^{1}$

\begin{abstract}
Purpose: The purpose of this study was to describe the phenotype (and corresponding genotype) of adult patients with late-onset Tay-Sachs disease, a clinical variant of the $\mathrm{G}_{\mathrm{M} 2}$-gangliosidoses. Methods: A comprehensive physical examination, including neurological assessments, was performed to establish the current disease pattern and severity. In addition, the patients' past medical histories were reviewed. The patients' $\alpha$-subunit mutations ( $\beta$-Hexosaminidase A genotype) were determined and correlated with their corresponding clinical findings and disease course. Results: Twenty-one patients (current mean age: 27.0 years; range: 14-47 years) were identified. The pedigree revealed a relative with the "classic" infantile or late-onset form of Tay-Sachs disease in four (out of 18) unrelated families. The patients were predominantly male (15/21 individuals) and of Ashkenazi Jewish ancestry (15/18 families). Mean age at onset was 18.1 years; balance problems and difficulty climbing stairs were the most frequent presenting complaints. In several cases, the diagnosis was delayed (mean age at diagnosis: 27.0 years). Analysis of the $\beta$-hex A gene revealed the G269S mutation as the most common disease allele; found in homozygosity $(N=1)$ or heterozygosity ( $N=18$; including 2 sib pairs). Disease onset (age 36 years) was delayed and progression relatively slower in the homozygous G269S patient. Two siblings (ages 28 and 31 years), of non-Jewish ancestry, were compound heterozygotes (TATC1278/W474C); their clinical course is dominated by psychiatric problems. Brain imaging studies revealed marked cerebellar atrophy in all patients $(N=18)$ tested, regardless of disease stage. Conclusions: Late-onset Tay-Sachs disease is an infrequent disorder and the diagnosis is often missed or delayed (by approximately 8 years). Early on, the majority of patients develop signs of either cerebellar or anterior motor neuron involvement. Affected individuals may also develop psychotic episodes. In most cases, the later-onset of expression results from the presence of at least one allele (usually the G269S mutation), associated with residual enzyme ( $\beta$-hexosaminidase A) activity. A positive family history is a valuable clue, enabling early diagnosis. Nonspecific cerebellar atrophy on brain imaging is another important finding. This entity should be considered among patients presenting with speech, gait, and balance problems, and those with psychiatric disorders even when focal neurologic deficits may be initially absent. Accurate diagnosis will permit appropriate genetic counseling regarding disease prognosis and reproductive risks. Genet Med 2005:7(2):
\end{abstract} 119-123.

Key Words: $G_{M 2}$ gangliosidosis, late-onset Tay-Sachs disease, $\beta$-hexosaminidase deficiency, G269S mutation, cerebellar atrophy.

The $\mathrm{G}_{\mathrm{M} 2}$-gangliosidoses represent a heterogeneous group of disorders resulting from a failure in lysosomal hydrolysis of $\mathrm{G}_{\mathrm{M} 2}$-ganglioside; due to a primary deficiency of the $\beta$-hexosaminidase A ( $\beta$-hex A) enzyme or its cofactor (the $\mathrm{G}_{\mathrm{M} 2}$ ac-

From Divisions of ${ }^{1}$ Neurogenetics and ${ }^{4}$ Neuropsychology, Department of Neurology, and the Departments of ${ }^{2}$ Pediatrics and ${ }^{3}$ Rehabilitation Medicine, New York University School of Medicine, New York, NY.

Edwin H. Kolodny, MD, Neurogenetics, NYU at Rivergate, 403 East 34th Street, 2nd floor, New York, NY 10016.

Received: July 23, 2004.

Accepted: October 12, 2004.

DOI: 10.1097/01.GIM.0000154300.84107.75 tivator protein). ${ }^{1}$ The $\beta$-hex A enzyme is a heteromeric protein comprised of an $\alpha$ - and $\beta$-subunit encoded by two separate genes (on chromosome 15 [q23-24] and 5 [q13], respectively). Mutations of the $\alpha$-subunit lead to $\beta$-hex A deficiency (the Tay-Sachs phenotype [TSD]), while gene defects involving the $\beta$-subunit result in a deficiency of $\beta$-hex A and B (Sandhoff disease). ${ }^{2}$ The $\mathrm{G}_{\mathrm{M} 2}$ activator is a nonenzymatic protein that mediates the interaction between the water-soluble enzyme $\beta$-hex $A$ and its membrane-embedded substrate, $\mathrm{G}_{\mathrm{M} 2 \text { - }}$ ganglioside, at the lipid-water interphase. Deficiency of the $\mathrm{G}_{\mathrm{M} 2}$ activator is referred to as the $\mathrm{AB}$ variant. ${ }^{3}$

Late-onset Tay-Sachs disease (LOTS) is a clinical subtype of the $\mathrm{G}_{\mathrm{M} 2}$-gangliosidoses, characterized by disease expression in 
childhood or later. In contrast to the classic infantile form of TSD, which usually leads to death between 3 and 5 years of life, patients with LOTS have a more protracted clinical course (Fig. 1). This variant is further classified into a juvenile-onset form typified by progressive spasticity and rigidity, with seizures and dementia; affected individuals ultimately enter a vegetative state between the ages of 5 and 15 years. ${ }^{4}$ Patients with the adult-onset form manifest in late childhood or in their teens (and in other cases much later), with signs of cerebellar (e.g., dysarthria, dysmetria, and ataxia) and anterior horn cell (e.g., proximal muscle weakness, fasciculations, and atrophy) involvement. In some patients, clinical presentation and disease course may be dominated by neuropsychiatric problems (e.g., bipolar disorder and depression). These clinical distinctions based on age at onset are arbitrary and represent the various modes of disease expression associated with deficient $\beta$-hex A activity. ${ }^{5}$ All of the $\mathrm{G}_{\mathrm{M} 2}$-gangliosidosis subtypes are inherited in an autosomal recessive fashion and the differences in age at onset and disease course may be partly attributed to the underlying $\beta$-hex A gene defect. Patients with infantile TSD tend to be homozygous or compound heterozygotes for severe, deleterious ("null") alleles, whereas patients with LOTS have a combination of one severe and another allele associated with residual $\beta$-hex A activity. ${ }^{6}$ These conditions are prevalent among individuals of Ashkenazi Jewish ancestry, although the introduction of a carefully structured screening program and detection and appropriate counseling of carrier couples at risk have reduced their incidence within this population. Rare cases of LOTS have been reported among non-Jewish people; often in association with private or unique alleles. ${ }^{7}$

We describe the clinical features at both the time of presentation and at diagnosis, and the current phenotype of 21 patients (from 18 families) with LOTS. In addition, genotype information is provided and correlated with the corresponding patient phenotype.

\section{MATERIALS AND METHODS}

The patients in this report were recruited to participate in a study of the natural history of LOTS, using a institutionalapproved protocol. After informed consent, the patients were asked detailed questions regarding their current problems and chief complaints at disease onset. During their initial clinic visit, each patient had a thorough physical examination, including an evaluation of their current neurologic status, and when feasible, an MRI of the brain. The diagnosis of LOTS was based on clinical findings and demonstration of decreased $\beta$-hex A activity in leukocytes or skin fibroblasts; from which DNA was also obtained for characterization of the patient's genotype (i.e., causal $\beta$-hex A mutation[s]). The enzyme assay and analysis of the $\beta$-hex $A$ gene sequence were performed using standardized methods (as previously described ${ }^{8}$ ).

Additionally, a review of the patients' medical records was performed based on availability, and information was also obtained from the parents when possible. This study was conducted between January 1, 2002 and December 31, 2003, and was partly supported by grants from the National Organization for Rare Disorders and Oxford GlycoSciencies, Ltd (UK).

\section{RESULTS}

During the study period, we identified 21 individuals (including three sib pairs) with LOTS, derived from 18 families (Fig. 1). The patients were predominantly male (15/21 [71.4\%] cases) and of Ashkenazi Jewish ancestry (15/18 families $[83.3 \%])$. Two of the three sib pairs identified and one addi-

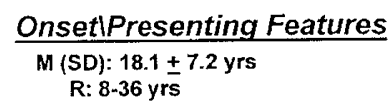

Onset|Presenting R: 8 -36 yrs
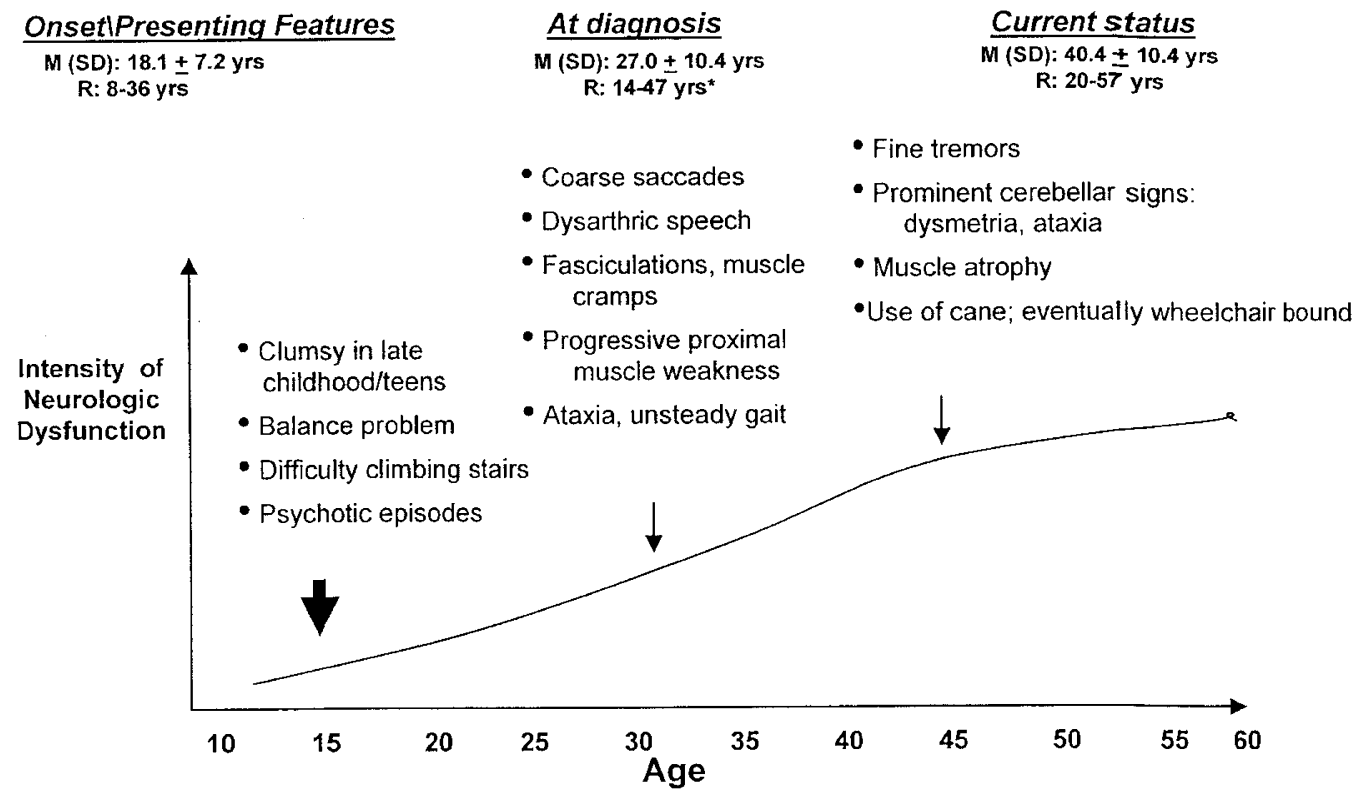

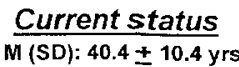

$M(S D): 40.4 \pm 10.4$ yrs

R: $20-57$ yrs

Fig. 1. LOTS: Clinical course and profile 
Table 1

$\beta$-hex A gene defects identified

\begin{tabular}{lc}
\hline Genotype & $N(\%)$ \\
\hline G269S/TATC1278 & $14(66.6)$ \\
G269S/IVS12 & $2(9.5)$ \\
G269S/R504C & \\
G269S/G269S & \\
TATC1278/W474C & \\
\hline
\end{tabular}

${ }^{a}$ Mutations identified in the non-Jewish study patients.

tional patient were Caucasians, but not of Jewish descent. A list of the patients' genotypes and their frequency distribution is provided in Table 1.

The patients' mean age ( \pm SD) at disease onset was 18.1 ( \pm 7.2) years (range: $8-36$ years). The majority of the patients recalled being clumsy in late childhood or in their teenage years, and had balance problems and progressive difficulty climbing stairs. At least five of the patients experienced psychotic episodes in their teens, for which an organic basis was suspected only in instances were focal neurologic deficits were found, or a positive family history was present (usually an older affected sibling as noted for three unrelated families); although the pedigree of one (other unrelated) family revealed the occurrence of a case of infantile TSD in a maternal first cousin.

The patients' mean age $( \pm \mathrm{SD})$ at diagnosis was 27.0 ( \pm 10.4) years (range: $14-47$ years). Findings noted around the time of diagnosis included coarse saccades, dysarthric "hot potato" speech, fasciculations (especially after physical exertion), bilateral proximal muscle weakness, and ataxia (with an unsteady gait). During this period, several patients also suffered from episodes of manic-depression, which in some cases was severe and required prolonged hospitalization. In a proportion $(N=17$ [81\%]) of the patients, the diagnosis of LOTS was delayed by about 8 to 10 years and the existing medical problems during consultation with local physicians were initially attributed to one of several other conditions, such as spinal muscular atrophy (the Kugelberg-Welander syndrome), muscular dystrophy (unspecified), amyotrophic lateral sclerosis, or spinocerebellar ataxia.

The current mean age ( \pm SD) of the patients is 40.4 ( \pm 10.4$)$ years (range: $20-57$ years). Clinical findings during their most recent evaluation (at study entry) revealed prominent cerebellar signs, with verbal communication problems and difficulties with writing and eating (primarily the need for assistance when cutting food). Two (9.5\%) patients require the use of a computerized device for information exchange. Most have some degree of muscle atrophy, and the majority (57.2\%) uses either a cane or walker $(N=6)$ or are wheelchair-bound $(N=6)$. The mean age $( \pm S D)$ for the group of latter patients, corresponding to the time when they became dependent on the wheelchair, was 41.0 ( \pm 7.2$)$ years.

In addition, on examination, several of the patients had fine tremors of the outstretched hands and easy startle to sudden light touch. Many patients have significant triceps muscle weakness, but arm strength is otherwise relatively well-preserved. Leg weakness was noted in all patients, especially in the iliopsoas and quadriceps muscles. The triceps reflex was greatly reduced or absent in all patients, and a crossed-adductor reflex was observed on tapping of the patellar tendon. None had major swallowing problems. Brain MRI obtained in 18 (86\%) patients revealed the uniform presence of marked cerebellar atrophy, regardless of disease stage. Neuropsychiatric assessments (reported separately ${ }^{9}$ ) performed in $17(81 \%)$ patients revealed that $30 \%-50 \%$ had delayed verbal recall and problems with cognitive processing, visual sequencing, and set shifting; none of these individuals had signs of significant global decline or dementia.

The mean $( \pm$ SD) duration of illness for the study population was 23.1 ( \pm 12.8 ) years. A review of the patients' individual course revealed disease progression was not linear and showed no correlation with either age at onset or genotype, with one exception. Onset of symptoms (at age 36 years) in this latter patient (currently age 54 years) was delayed and disease course was more protracted. This individual is of Polish-Austrian ancestry and homozygous for the G269S mutation, as previously reported. ${ }^{7}$ Until about 5 years ago, the patient was able to ambulate independently. At present, he is able to transfer without assistance and is able to walk over a short distance using a cane for balance; however, the use of a wheelchair is required to get around in public. Furthermore, in contrast to the other patients his speech is fluent and coherent and there is minimal past-pointing on finger following. The patient has also not suffered from any major psychiatric problems. Despite these relatively milder findings, brain MRI revealed cerebellar atrophy which is indistinguishable from that noted in the other patients.

Two individuals (non-Jewish sib pair, of Canadian-Portuguese and German-Dutch ancestry) are compound heterozygotes for TATC1278/W474C ( $\alpha$-subunit mutations of the $\beta$-hex A gene, which represent, respectively, a null allele and missense mutation associated with residual enzyme activity). They had mild speech and gait problems at onset (between 12 and 13 years). The diagnosis of LOTS in this family was made after the older sibling (age 16 years at the time) experienced a severe psychotic episode (characterized by marked social and emotional withdrawal, paranoia, obsessive thinking, and compulsiveness associated with marked agitation). Currently, both siblings (age 28 and 31 years) have an exceptional disease course dominated by psychiatric problems; they are withdrawn and do not engage in social interaction, and speech (utterance) is limited but understandable without the need for repetition. Although both patients are able to ambulate independently they are greatly dependent on assistance from their parents with most routines of daily living (e.g., they will not eat or groom themselves unless guided).

Two additional individuals (also non-Jewish sib-pair, of Lithuanian-Scandinavian and German-Polish descent) are compound heterozygotes for the G269S/R504C mutations ( $\alpha$ subunit defects of the $\beta$-hex A gene that represent missense 
mutations associated with [G269S] and without [R504C] residual enzyme activity, respectively). Their diagnosis was made at the ages of 23 and 25 years; in the latter patient (and the younger of the two) in the context of the known family history and after a major psychotic episode. This individual became confused and paranoid while at work and required hospitalization. Both siblings reported not being active in sports since their mid-teens because they "felt awkward." Currently, they have moderate dysarthria and dysmetria (of similar degree). The older sibling is 40 years old and has a slightly more pronounced gait problem necessitating the use of a walker to ambulate. The younger individual (age 32 years) is able to walk independently. Both are fully employed. These patients appear to be following a similar disease course, and the difference in degree may be accounted for by the age discrepancy.

Disease expression in the other patients with other genotypes (i.e., $\beta$-hex A $\alpha$-subunit gene mutations) is indistinguishable from the described individuals who were compound heterozygotes for the G269S/R504C mutations. Although a significant proportion $(18 / 21$ [85.7\%]) of the patients have psychiatric problems, these are well stabilized by medications and not as incapacitating and persistent as that observed in the siblings with the TATC1278/W474C mutations.

\section{DISCUSSION}

Late-onset Tay-Sachs disease (LOTS), a clinical subtype of the $\mathrm{G}_{\mathrm{M} 2}$-gangliosidoses, is characterized by a combination of features resulting from involvement of the cerebellum (e.g., dysarthria, dysmetria, and ataxia) and anterior horn cell (e.g., proximal muscle weakness and atrophy). Disease manifestations may be apparent from early or late childhood, but in some cases, significant problems may not develop until the patients are in their teens or in adulthood; and the diagnosis can be initially missed, particularly among non-Jewish individuals and when the family history is noncontributory. As LOTS is an autosomal recessive trait, delayed diagnosis may lead to the inability to provide appropriate genetic counseling regarding reproductive risks, particularly in the high-risk populations such as the Ashkenazi Jewish (AJ). LOTS is a rare disorder, with an estimated incidence in the AJ population of 1 in 135,000; the incidence in the non-Jewish population is unknown but may approximate that of infantile Tay-Sachs or Sandhoff disease $(\approx 1$ in 300,000$)$ in this group. It is possible that these figures may represent an underestimate, particularly among patients with atypical presentations, slowly progressive ataxia or mild speech impediments.

Early findings in patients with LOTS include decreased or absent triceps and patellar reflexes and cerebellar atrophy on brain MRI. Additional observations include an easy startle, primarily to light touch when unexpected, hand tremors, fasciculations, and a positive palmomentum reflex (i.e., contraction of the mentis muscle causing wrinkling of the chin with elevation and protrusion of the lower lip when the skin over the thenar eminence is scratched, also known as the MarinescoRadovici sign).
Analyses of the $\beta$-hex A gene for mutations within the $\alpha$-subunit among affected patients reveal the frequent occurrence of the G269S mutation (resulting from a G to A transition in exon 7). This particular defect accounts for approximately two percent of $\mathrm{G}_{\mathrm{M} 2}$-gangliosidosis disease alleles among individuals of AJ descent. ${ }^{6}$ In our study of LOTS patients, the G269S allele was found in 59\% (18/44) of the mutation-bearing chromosomes. This particular mutation, which is associated with residual enzyme activity (based on in vitro expression studies), was usually found in combination with a deleterious allele such as the null mutations TATC1278 or $\mathrm{IVS}_{12}{ }^{+1}$. In AJ and non-Jewish patients with the "classic" infantile TSD, the TATC1278 mutation (i.e., a frameshift defect resulting from a 4 base insertion in exon 11) accounts for $81 \%$ and $8 \%$ of disease alleles, respectively. The IVS $12^{+1}$ mutation (i.e., a splicing error resulting from a $\mathrm{G}$ to $\mathrm{C}$ substitution in intron 12) has only been reported among the AJ patients, in whom it accounts for $9 \%$ of disease alleles.

Two additional $\beta$-hex A $\alpha$-subunit mutations (W474C and R504C) were identified in two of our study patient's families (in combination with the TATC1278 and G269S mutation, respectively). The W474C $1422 \mathrm{G}$ to C (a nucleotide substitution in the first position of exon 13 of the $\alpha$-subunit $\beta$-hex A) found in one of the non-Jewish families has been previously reported. ${ }^{10}$ Transient coexpression studies of this defect with the normal $\beta$-subunit gene sequence to produce $\beta$-hex $\mathrm{A}$ in COS-7 cells revealed the presence of mature $\alpha$-subunit. However, the level of expressed protein was much lower than that from normal $\alpha$-subunit transfections; although higher than in those cells transfected with an $\alpha$-subunit mutation (such as TATC1278) associated with the infantile TSD. These residual findings may explain the later (nonclassic) presentation of the involved patients, in whom the W474C mutation was found in combination with a null allele (TATC1278). The R504C mutation (in combination with another allele) has also been described previously. ${ }^{11}$ Expression of the mutagenized cDNA for this allele with a cysteine substitution gave rise to an $\alpha$-subunit that was secreted primarily as the $\alpha$-monomer (rather than as the normally expected $\alpha \alpha$ dimer); the corresponding protein was associated with no enzymatic activity. Thus, in this individual the residual activity resulting from the G269S mutation was sufficient to transform the phenotype from the classic infantile to the late onset form. This supposition is also supported by the relatively milder and later onset of disease in the patient homozygous for the G269S mutation.

Psychiatric problems (e.g., acute psychosis associated with marked disorganization of thought, agitation, delusions and hallucinations, mania and paranoia, as well as recurrent psychotic depression) are frequent among patients with LOTS. ${ }^{12,13}$ The anatomic bases of these deficits and other abnormalities in executive functioning and memory (exhibited by a subset of affected individuals) are not well understood. The use of traditional neuroleptics to treat the psychosis in these individuals may produce adverse outcomes. ${ }^{14}$ In the case of tricyclic antidepressants and phenothiazines, the poor response to treatment has been attributed to the observation that 
these drugs inhibit $\beta$-hex A activity in vitro and induce lysosomal lipidosis in fibroblasts and accumulation of lipids in experimental animals in vivo. Treatment with lithium salts and electroconvulsive therapy has been reported to be beneficial, ${ }^{15}$ at least in ameliorating the episodes of psychotic depression. Thus, recognition of the diagnosis and familiarity with the types of medications to avoid is critical in the overall management of symptomatic patients.

Brain imaging studies in patients with LOTS generally show severe cerebellar atrophy. Similar to the findings reported previously, ${ }^{16}$ we found no correlation between disease severity and degree of cerebellar atrophy.

In summary, the diagnosis of late-onset Tay-Sachs disease should be considered among patients presenting with speech, gait, and balance problems, and those with psychiatric disorders even when focal neurologic deficits may be initially absent. Accurate diagnosis will permit appropriate genetic counseling regarding disease prognosis and reproductive risks.

\section{References}

1. Gravel RA, Clarke JTR, Kaback MM, Mahuran D, Sandhoff K, Suzuki K. The GM2 gangliosidoses. In: Scriver CR, Beaudet AL, Sly WS, Valle D, eds. The metabolic and molecular bases of inherited disease. New York: McGraw-Hill, 1995:2839-2882.

2. Mahuran DJ. The biochemistry of HEXA and HEXB gene mutations causing GM2 gangliosidosis. Biochim Biophys Acta 1991;1096:87-94.

3. Mahuran DJ. The GM2 activator protein, its roles as a co-factor in GM2 hydrolysis and as a general glycolipid transport protein. Biochim Biophys Acta 1998;1393:1-18.

4. Matsuzawa F, Aikawa S, Sakuraba H, Lan HT, Tanaka A, Ohno K, Sugimoto Y, Ninomiya H, Doi H. Structural basis of the GM2 gangliosidosis B variant. J Hum Genet 2003;48:582-589.
5. Neudorfer O, Kolodny EH. Late-onset Tay-Sachs disease. Isr Med Assoc J 2004;6: 107-111.

6. Paw BH, Kaback MM, Neufeld EF. Molecular basis of the adult- onset and chronic GM2 gangliosidosis in patients of Ashkenazi Jewish origin: substitution of serine for glycine at position 269 of the $\alpha$ subunit of $\beta$-hexosaminidase. Proc Natl Acad Sci USA 1989;86:2413-2417.

7. Navon R, Kolodny EH, Mitsumoto H, Thomas GH, Proia RL. Ashkenazi-Jewish and non-Jewish adult GM2 gangliosidosis patients share a common genetic defect. Am J Hum Genet 1990;46:817-821.

8. De Gasperi R, Gama Sosa MA, Battistini S, Yeretsian J, Raghavan S, Zelnik N et al. Late-onset GM2 gangliosidosis: Ashkenazi Jewish family with an exon 5 mutation (Tyr180-> His) in the Hex A alpha-chain gene. Neurology 1996;47:547-552.

9. Zaroff CM, Neudorfer O, Morrison C, Pastores GM, Rubin H, Kolodny EH. Neuropsychological assessment of patients with late onset GM2 gangliosidosis. Neurology 2004;62:2283-2286.

10. Petroulakis E, Cao Z, Clarke JT, Mahuran DJ, Lee G, Triggs-Raine B. W474C amino acid substitution affects early processing of the alpha-subunit of beta-hexosaminidase A and is associated with subacute G(M2) gangliosidosis. Hum Mutat 1998;11: 432-442.

11. Paw BH, Wood LC, Neufeld EF. A third mutation at the CpG dinucleotide of codon 504 and a silent mutation at codon 506 of the HEX A gene. Am J Hum Genet 1991;48:1139-1146.

12. Rosebush PI, MacQueen GM, Clarke JT, Callahan JW, Strasberg PM, Mazurek MF. Late-onset Tay-Sachs disease presenting as catatonic schizophrenia: diagnostic and treatment issues. J Clin Psychiatry 1995;56:347-353.

13. Streifler J, Golomb M, Gadoth N. Psychiatric features of adult GM2-gangliosidosis. Br J Psychiatry 1989;155:410.

14. Filho JAF, Friedman SH, Kyle EA, Shapiro B. Medication Survey in Patients with Hexosaminidase A Deficiency: Implications for Treatment. Neurology 2003; 60(suppl 1):A52.

15. Renshaw PF, Stern TA, Welch C, Schouten R, Kolodny EH. Electroconvulsive therapy treatment of depression in a patient with adult GM2-gangliosidosis. Ann Neuro 1992;31:342.

16. Streifler JY, Gornish M, Hadar H, Gadoth N. Brain imaging in late-onset GM2 gangliosidosis. Neurology 1993;43:2055. 